\title{
Implementation of Algorithms of Image Analysis in the Software Package ColourUnique PRO with the Aim of Increasing the Accuracy of Classification Types Individuals
}

\author{
I.S. Tarasova ${ }^{1}$, A.V. Chechin ${ }^{1}$, V.V. Andreev ${ }^{2}$ \\ tar06@list.ru|chechin-a@yandex.ru|vyach.andreev@mail.ru \\ ${ }^{1}$ Nizhny Novgorod State University of Architecture and Civil Engineering, Nizhny Novgorod, Russia; \\ ${ }^{2}$ Nizhny Novgorod State Technical University n. a. R. E. Alekseev, Nizhniy Novgorod, Russia
}

\begin{abstract}
This paper consider the possibilities and prospects of the ColorUnique Pro software complex as a tool for automating the "Associative color space" $\mathbb{C}$ method of professional orientation testing and improving the accuracy of classification of test forms representing color images. The authors provided the data of verification and testing of the methodology, reflect the relevance of the study and justify the need to translate the testing into the format of the software package. In the process of implementation of the program complex, has been identified a problems, proposed solutions and prospects for the development of the program. Now, the authors have successfully optimized the test form, significantly reduced the time of testing, created a color palette that facilitates evaluation and classification of the final color images.
\end{abstract}

Keywords: career guidance, software package, optimization, analysis, classification, matrix, colour.

\section{Introduction}

The development of career guidance involves the actualization of classical methods (for example, the Holland's Career Test) and the development new, in accordance with the needs of employers and young recipients.

The Department of Industrial design of the Russian Nizhny Novgorod State University of Architecture and Civil Engineering, has been developed the career testing method «The associative color space» (C). This method, developed by

I. S. Tarasova, allows to classify recipients according to individual style of activity [2].

The method involved making the developed test form quasispace with color manually, by means of water-soluble paint or markers (Fig. 1, a). The obtained images are correlated by the expert (educational psychologist) with the standard models, derived by observing the group of experts of the development of recipients as students and in the future - as specialists. The standards are the most typical samples for 5 years. The group of experts consists of teachers, psychologists and heads of practice of recipients [5].

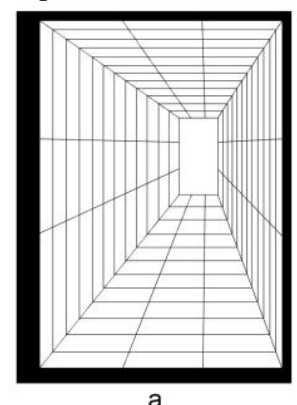

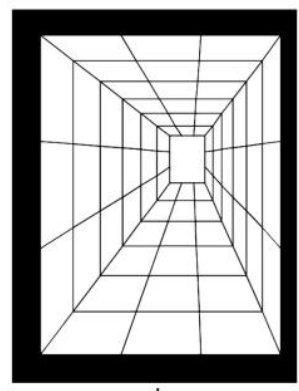

Fig. 1. The test form quasi-space: a. - basic, b. - optimized

By observation and experiment, derived five types of individuals by the style of pond activity. They are «creator», «rational», «skeptic», «avant-gardist» and «mixed type» (Fig. 2). There are subtypes: «cold rational», «warm rational», «noncontrast creator», «contrast creator», «skeptical the creator», «non-radical avant-gardist», «radical avant-gardist», «nonsaturated sceptic» and «saturated sceptic» [4].

Characteristic of a particular type of methods of arrangement of dark and light areas were derived by a group of experts by observation, the conclusions about the nature of the recipient's color preferences allows us to make the theory of Johannes Itten, set out in the work «the Art of color» [3].

The developed technique distinguished by the possibility of providing a more flexible and widespread recommendation to the recipient, as the derived types are found among both «techies» and «humanitarians».

But manual making of the test, both in class and at home, takes too much time (up to 3 hours), the basic form quasi-space (Fig. 1, a) contains a lot of lines, which makes it difficult for visually impaired recipients and people with no artistic experience. In addition, classical techniques (for example, the Holland's Career Test and Lucher test) are translated into computer program format and available on specialized sites, which makes them accessible and convenient for a wide range of recipients, not only designers and artists [6].

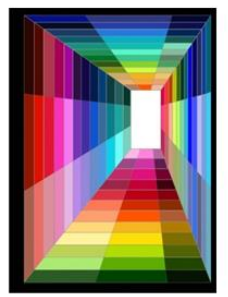

a.
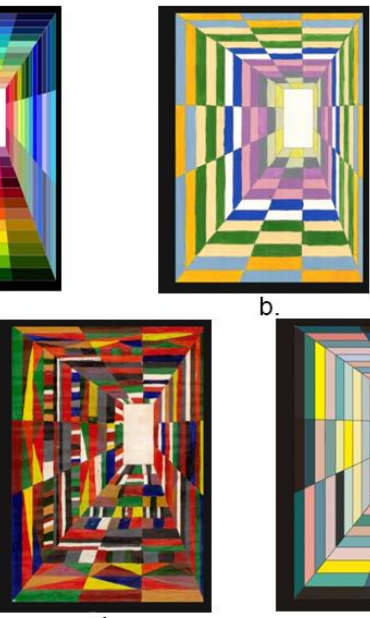

d.

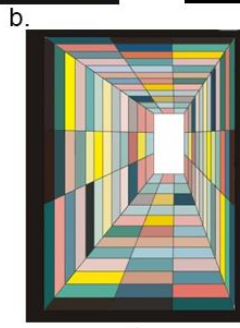

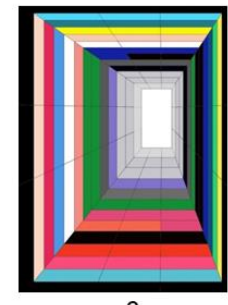

c.

Fig. 2. The types of individuals by the style of pond activity: a. - «creator», b. - «rational», c. - «skeptic», d. - «avant-gardist», e. - «mixed type»

So, for more verification and refinement of the classification criteria, the method of testing "Associative color space» (C) needed to optimize the basic test form quasi-space and transfer the test to the format of the software package ColourUnique Pro with the development of image evaluation algorithms and their classification in accordance with the derived standards (types of individuals). 
The software package is developed by I. S. Tarasova, A. V. Chechin and A. V. Shapoval, and includes the following software modules:

ColourUnique $\mathbf{M},-$ where $\mathrm{M}$ is monitoring. The first part of the program, which collects and systematizes data about the user (age, gender, occupation, level of education), as well as the automation of the process of filling out the test form. Readymade forms are still being evaluated by a teacher or psychologist.

ColourUnique A, - where $\mathrm{A}$ is the analysis. This program already includes a number of algorithms for correlating test forms with the derived types of individuals, the process is still controlled by a person, evaluates the correctness of the program, debugs and formulates information about errors, if there are errors.

ColourUnique $\mathbf{R}$, - where $\mathrm{R}$ is the result. At this stage, the program automatically generates detailed recommendations and displays the test result in a PDF file available for download on a PC or smartphone. The task of a person is to assess the correctness of the recommendations, their volume and ease of download, as well as reading the file from the screen of a PC or smartphone.

ColourUnique Pro - final software system, and carrying out data collection, and analysis and evaluation. This version should ideally exclude the participation of a psychologist.

\section{Verification}

All recipients tested by the method of «Associative color space» (C) since 2016, have been tested in parallel by the method of Holland. The Holland's method is a test that contains a pair of professions between which the recipient is asked to choose the most preferred. As a result, points are recruited that relate to a particular type of focus and preferences of the recipient. There are 6 of them. They are «realistic», «investigative», «artistic», «social», «enterprising» and «conventional».

It is important, that when calculating the results in this control group from the histogram excluded scale «artistic», because designers and artists in the group has this scale like the dominant in $90 \%$ of cases.

The results of data comparison and correlation search are presented on the histogram (Fig. 3).

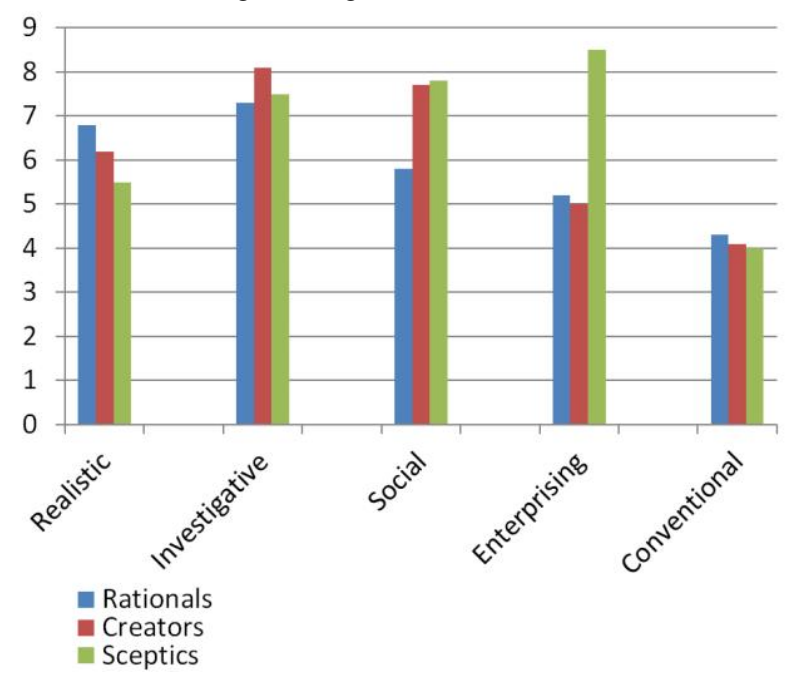

Fig. 3. The histogram of distribution of points according to the scales of Holland's Career Test

As can be seen from the histogram, recipients of the «skeptic» type gain significantly more points on the scale of preferences of the «entrepreneurial» type, namely 8.5 points against the same indicator of «rationals» count to 5.2 and «creators» count to 5 .
The social scale of «creators» and «skeptics», equal to 7.7 and 7.8 respectively, exceeds the same indicator of «rationals», which is 5.8 points.

«Rationals» also show a higher rate on the scale of «realistic», which is 6.8 , while the rate of «creators» is 6.2 , and «skeptics»- 5.5 points.

In the future, development team plans to conduct a similar study for the groups of «social», «enterprising», «investigative» and other areas, with the exception of the corresponding dominant indicator, if such is found as a result of the experiment.

\section{Optimization and algorithms of the software package ColourUnique Pro}

Experimentally, a group of developers found the optimal number of lines for the formation of quasi-space, equal to 6 (Fig. 1, b.) Such quantity allows to reproduce any characteristic structure of the accepted standards.

So, «creators» prefer to use a large number of shades and gradients, «rationals» distribute dark and light spots on the «chess» principle, «skeptics» - on the «linear» principle, «radical avant-gardist» - the only type that complements the original quasi-space figures and lines (Fig. 2).

First, let's consider the translation of the developed test form of quasi-space into digital form. In the software module name ColourUnique $\mathrm{M}$, this is implemented by matrices:

1. Base points of the outer and inner rectangle:

$$
\begin{gathered}
\text { OutBox }=\left|\begin{array}{ll}
P o b_{11} & P o b_{12} \\
P_{0} b_{21} & P o b_{22}
\end{array}\right|, \\
\text { InnerBox }=\left|\begin{array}{ll}
P i b_{11} & P i b_{12} \\
P i b_{21} & P i b_{22}
\end{array}\right| .
\end{gathered}
$$

2. Matrix of diagonals, after splitting:

$$
\text { Diag }=\left|\begin{array}{lllllll}
P d_{11} & P d_{12} & P d_{13} & P d_{14} & P d_{15} & P d_{16} & P d_{17} \\
P d_{21} & P d_{22} & P d_{23} & P d_{24} & P d_{25} & P d_{26} & P d_{27} \\
P d_{31} & P d_{32} & P d_{33} & P d_{34} & P d_{35} & P d_{36} & P d_{37} \\
P d_{41} & P d_{42} & P d_{43} & P d_{44} & P d_{45} & P d_{46} & P d_{47}
\end{array}\right| \text {, }
$$

where: $P d_{11}=\operatorname{Pob}_{11}, P d_{21}=\operatorname{Pob}_{12}, \ldots$

$$
\ldots P d_{17}=P i b_{11}, P d_{27}=P i b_{12}, \ldots
$$

3. Matrix of parties in one of four directions, after breakdown:

$$
S=\left|\begin{array}{cccc}
P s_{11} & P s_{12} & P s_{13} & P s_{14} \\
P s_{21} & P s_{22} & P s_{23} & P s_{24} \\
\cdots & \cdots & \cdots & \cdots \\
P s_{71} & P s_{72} & P s_{73} & P s_{74}
\end{array}\right|,
$$

where: $P s_{11}=P d_{11}, P s_{14}=P d_{21}$ and so on.

4. Matrix of cells in one of four directions:

$$
\text { Cells }=\left|\begin{array}{ccc}
C_{11} & C_{12} & C_{13} \\
C_{21} & C_{22} & C_{23} \\
\ldots & \ldots & \ldots \\
C_{61} & C_{62} & C_{63}
\end{array}\right|
$$

where each cell consists of side points:

$$
C_{11}=\left|\begin{array}{ll}
P s_{11} & P s_{12} \\
P s_{21} & P s_{22}
\end{array}\right|
$$


Cell location coordinates $(C)$ have the following relationship:

$$
C=f(P ; k ; B ; D ; S),
$$

where $P$ - the coordinates of the base points of the outer rectangle, $k$ - the cells compression ratio, $B$ - the block of the quasi-spatial model, $D$ - the diagonal, $S$ - the sides.

The stages of generation of cells:

1. The initial specifying parameters, generation of base points and calculation of proportions;

2. The creation of quadrangular cells, including the proportional breakdown of the diagonals and sides;

3. The entry of cells into the database.

The development of the ColourUnique Pro software package includes the use of Internet technologies. This is necessary in order to organize multi-user testing and collection of results into a single database. On the server side, Python is used to calculate point coordinates [1].

The first thing the user sees when opening the program is an input questionnaire. It is needed to collect statistics and send the result to the recipient:

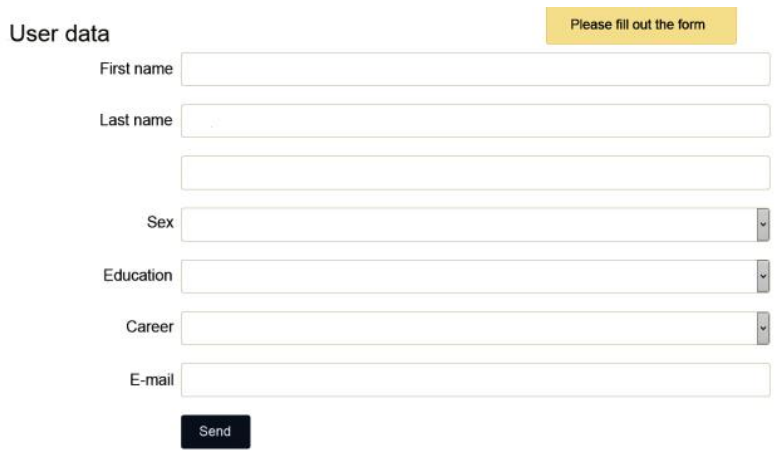

Fig. 4. The form of user data collection in the software package ColourUnique Pro

After filling out the form, the recipient enters the program interface, where the test form is located. Sounds audio instruction, at the end of which the menu becomes active:

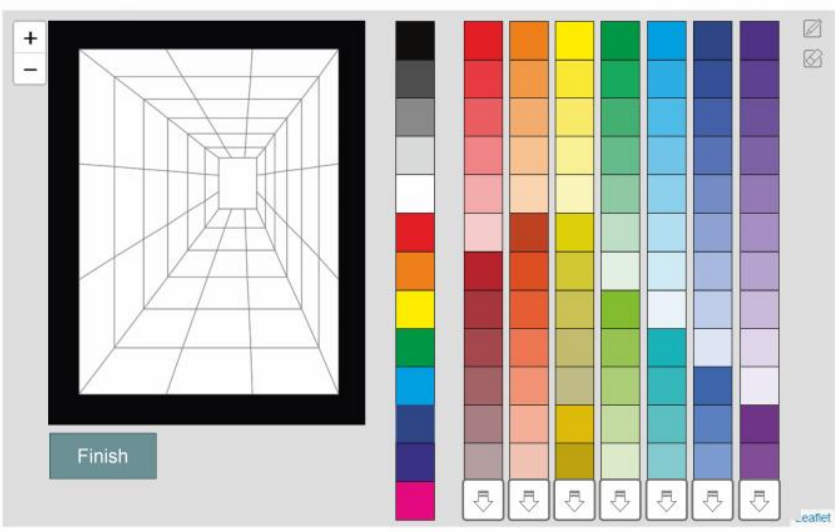

Fig. 5. The interface of the software module ColourUnique Mthe first module of the software package ColourUnique Pro

The task of the recipient is to fill in the form with the colors offered in the graphical editor in accordance with the Association specified by the voice instructor. At the end of the work with the form, the recipient presses the «Finish» button. Next, the program analyzes the resulting image in accordance with the specified algorithms.

Before the implementation of the software, the algorithm had the appearance as shown on Fig. 6.

In the process of realization, the first stage (Phase 1) of the algorithm was implemented - after its passage with 100 percent probability, the recipients related to the subtype «radical avant- gardist» are identified. This is due to the fact that only this subtype in the process of passing the test uses the tools «Pencil» and «Eraser».

This is followed by Phase 2 - analysis of the color profile of the recipient.

Consider in more detail on the review of the graphical editor of the ColourUnique Pro software package.

The fact that the graphic editors of programs such as, for example, Adobe Photoshop, built on the display of chromatic colors in RGB, CMYK, Indexed colors and Lab modes.

For the designer, this gives excellent opportunities to obtain the most complex and diverse shades, however, in the case of assessing the color profile of the recipient software complex color unique, there is a problem of correlation shades close to gray, or composite shades with belonging to a particular tone. For example, «red», «orange», «yellow» and so on.

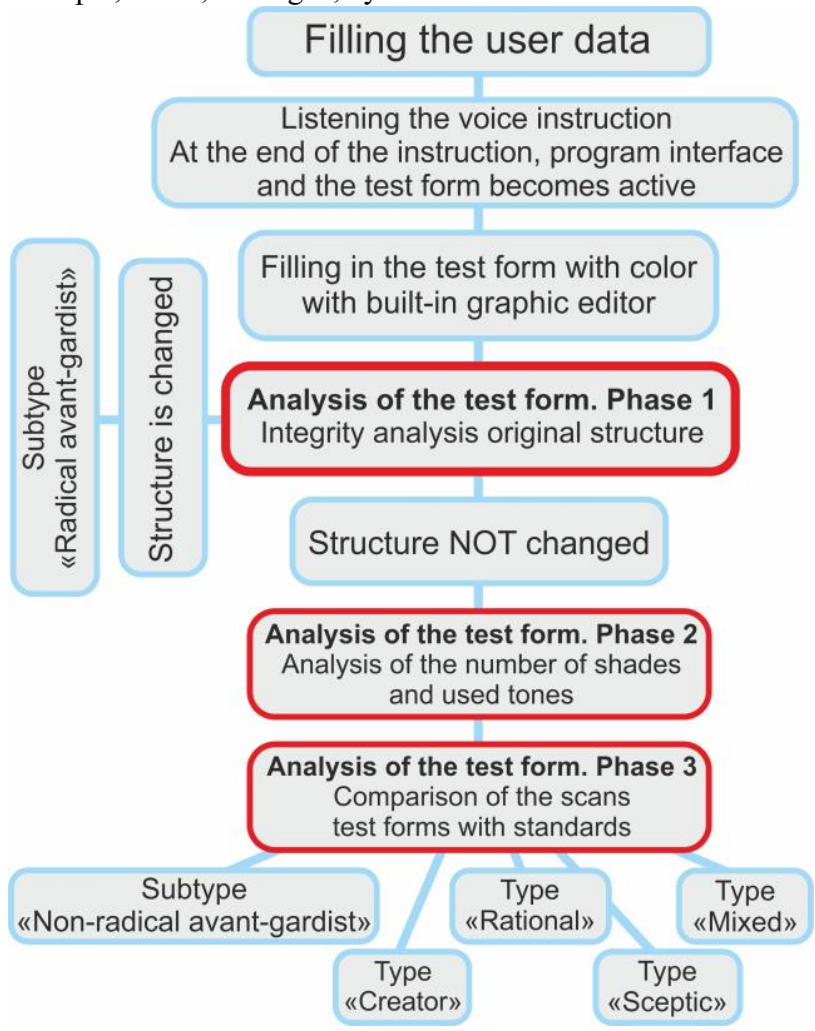

Fig. 6. The initial algorithm of analysis and classification of test forms

Based on the RAL color system, the group of developers selected the colors indicated in the system as shades of «red», «orange» and so on [7].

Thus, the recipients are offered ready-made pallets, the coordinates of the shades of which are already recorded in the program code as colors related to a specific tone. The "creators", prone to creating gradients, will be able to use ready-made stretch marks, other types will find in the pallets as the main colors and shades of gray.

This step allowed not only to reduce the time of filling the test (from 3 hours manually to $15-40$ minutes), but also to significantly improve the original type recognition algorithm.

The Fig. 7 shows the distribution of «creators», «rationals» and «skeptics» by the total number of colors used. The subtype "non-radical avant-gardist" is not added to this sample, as at the moment only $4 \%$ of the total sample for 5 years of testing are representatives of such type.

The diagram shows that representatives of the «creator» type use a much greater number of colors and shades, «rationals» and «skeptics». Thus, the average total number of 
shades of «creators» has 37 points, «rationals» - 12 points, «skeptics»- 9 points.

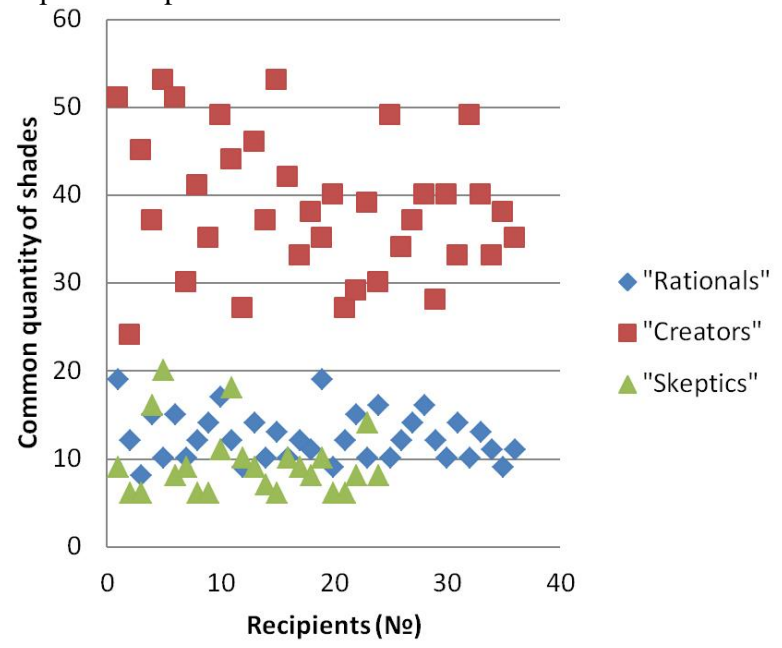

Fig. 7. The distribution diagram of the types «creator», «rational» and «skeptic» by the total number of colors used

It is important to note that the program considers cells filled with colors that have the same RGB coordinates in one used shade. This distribution result is explained by the feature of "creators» to create gradients, which involves the use of a large number of shades.

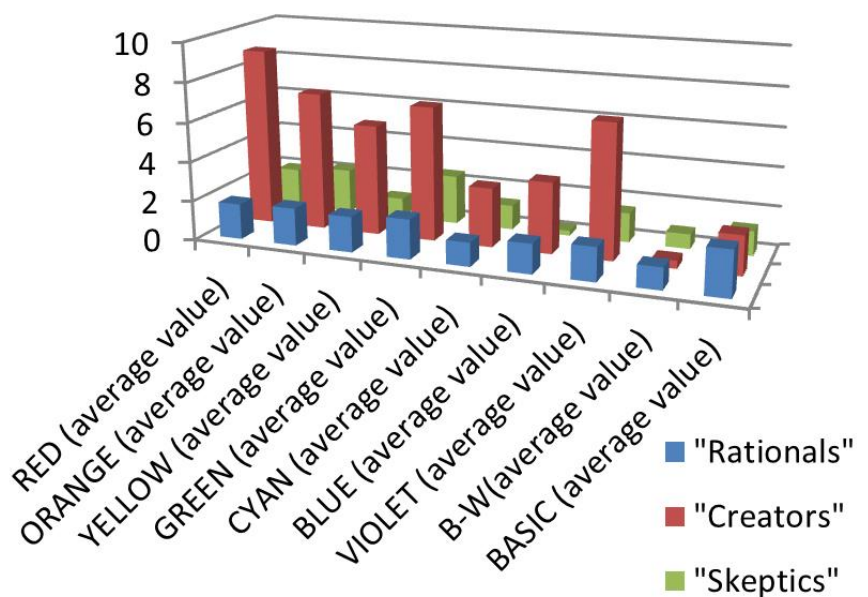

Fig. 8. The histogram of the average number of shades of each tone of the types «creator», «rational» and «skeptic»

Consider the histogram shown in fig. 8. This histogram most clearly characterizes how much more different shades are used on average the «creators» in comparison with other types. In this group of creators there are both their subtypes - contrast and noncontrast. The most preferred tone of «creators» - red, the least shades of gray. «Rationals» tend to be about the same across the spectrum, «skeptics»- to reds-oranges and greens.

The stage of color analysis (Phase 2) allows you to select the type of «creator» with high accuracy. The «rational» and «skeptic» types also show some color preferences, however, they are not enough to accurately separate these two types at the second stage of the original algorithm.

At the moment, the developers are considered to be the recipient of the "creator», if its color profile at least one tone, there are 5 or more shades.

\section{Conclusion}

At the moment, the test form quasi-space has been successfully converted into an electronic format, reduced from 12 to 6 lines, oriented in such a way that it is convenient for the user to fill each sector, despite the perspective distortion.

The testing procedure is significantly simplified and reduced, on average, from 3 hours to 15-40 minutes (the Creator fills out

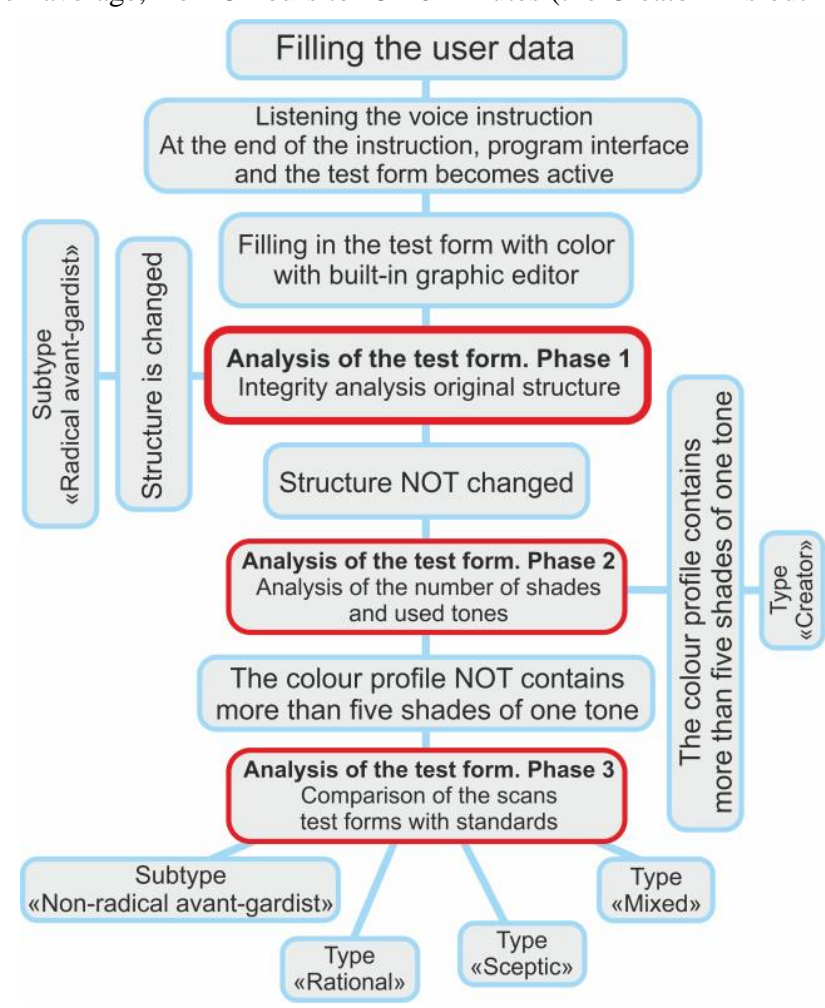

the form longer)

The simplified graphic editor allowed to analyze the simplified test forms and highlight the quantitative characteristics characteristic of the creators (from 5 shades of one tone, the total number of colors used on average 37 points).

The original algorithm is refined (Fig 9).

Fig. 9. The refined algorithm of analysis and classification of test forms

To distinguish the types of «rational», «skeptic» and subtype «non-radical avant-gardist» (Phase 3), an algorithm for recognizing the «chess» and «linear» color distribution is developed, since «non-radical avant-gardists» are also characterized by the formation of lines, but vertical (in the case of a «skeptic» - horizontal lines).

The study shows that it is possible to derive a specific "filter" for each type.

The program module ColourUnique $\mathrm{M}$ is implemented and the program module ColourUnique $\mathrm{A}$ is started.

The development team plans to introduce associative networks into the analysis and classification process (for example, Hopfield networks).

\section{Gratitudes}

The work was partially supported by RFBR, Grant № 19-0700455 .

Speaker I. S. Tarasova expresses gratitude for the help in carrying out this research to the head of the Department of Industrial design of Nizhny Novgorod State University of Architecture and Civil Engineering - A.V. Shapoval, the head of the Department «Nuclear reactors and power plants» of Nizhny 
Novgorod state technical university n. a. R. E. Alekseev, V. V. Andreev, docent of the Department of Geoinformatics, geodesy and cadastre of Nizhny Novgorod State University of Architecture and Civil Engineering A. V. Chechin, and academic groups D 29.16, D 30.17, and D 31.18 the Department of Industrial design of Nizhny Novgorod State University of Architecture and Civil Engineering for participation in scientificresearch work.

\section{References}

[1] Chechin, A. V. Processing of GEODATA using the Python language $[$ Text] / V. A. Chechin // proceedings of the scientific Congress of the 20th International scientific-industrial forum "Great Rivers 2018" - N. Novgorod: NNGASU. - P. 379-380.

[2] Grigorieva, M. V. Psychology of work. Lecture notes [Text] / M. V. Grigorieva. - Moscow: Higher education, 2006. - 192 p. [3] Itten, J. The art of color, I. Itten; TRANS. with it. and Foreword. L. Monakhova. - 2nd ed. - M. : D. Aronov, 2001. 95 p.: Il. - ISBN 5-94056-003-2.

[4] Tarasova, I. S. Prediction of the distribution of the visual mass of the dominant color combinations based on the analysis of the quasi-space model [Text] / I. S. Tarasova, V. V. Andreev, E. A. Martemyanova // Journal of Scientific and technical Bulletin of the Volga region, №5, 2018. - Pp. 256-260.

[5] Torshilova, E. M. Can you believe harmony with algebra? : Critic. the essay is expert. aesthetics / E. M. Torshilova. Moscow : Art, 1989. - 207,[1] p.; $20 \mathrm{~cm}$. Experimental aesthetics FB 2 89-19/310, FB 2 89-19/311

[6] CareerGuidance.ru [Electronic resource]: Website: https://proforientator.ru/

[7] Ral.ru [Electronic resource]: Website: https://ral.ru 